This item was submitted to Loughborough's Research Repository by the author.

Items in Figshare are protected by copyright, with all rights reserved, unless otherwise indicated.

\title{
On the role of nonlinear distortion in the theory of wave-like aquatic propulsion
}

PLEASE CITE THE PUBLISHED VERSION

https://doi.org/10.1016/j.oceaneng.2017.08.049

\section{PUBLISHER}

(C) Elsevier

\section{VERSION}

AM (Accepted Manuscript)

\section{PUBLISHER STATEMENT}

This work is made available according to the conditions of the Creative Commons Attribution-NonCommercialNoDerivatives 4.0 International (CC BY-NC-ND 4.0) licence. Full details of this licence are available at: https://creativecommons.org/licenses/by-nc-nd/4.0/

\section{LICENCE}

CC BY-NC-ND 4.0

\section{REPOSITORY RECORD}

Krylov, Victor V.. 2017. "On the Role of Nonlinear Distortion in the Theory of Wave-like Aquatic Propulsion". figshare. https://hdl.handle.net/2134/26464. 


\title{
On the role of nonlinear distortion in the theory
}

\section{of wave-like aquatic propulsion}

\author{
V.V. Krylov* \\ Department of Aeronautical and Automotive Engineering, \\ Loughborough University, \\ Loughborough, Leicestershire, LE11 3TU, UK
}

\begin{abstract}
Recent experimental investigations of small model boats propelled by propagating flexural waves carried out by the present author and his co-workers demonstrated viability of this type of propulsion as an alternative to a well-known screw propeller. Since the amplitudes of propagating flexural waves propelling the model boats are large enough, it is natural to consider the effect of nonlinear distortion of propagating localised flexural waves on generated thrust. This problem is explored in the present work by adding nonlinear harmonics of propulsive flexural waves to the well-known Lighthill's formula for generated thrust, which predicts a zero value of thrust in the case of linear flexural wave of constant amplitude. For simplicity, only the lowest (third) harmonic growing linearly with the distance of propagation is used. The resulting formula for the averaged thrust shows that, due to the effect of the third harmonic, the thrust is no longer zero, thereby demonstrating that nonlinear distortion of the propulsive flexural waves contributes positively to the generated thrust.
\end{abstract}

\footnotetext{
* Corresponding author. Tel.: +44 1509 227216; E-mail address: V.V.Krylov@lboro.ac.uk (V.V. Krylov)
} 
Keywords: Wave-like aquatic propulsion; Localised flexural waves; Hydrodynamic thrust; Elastic nonlinearity; Nonlinear distortion.

\section{Introduction}

It is well known that the most common method of aquatic propulsion used in existing marine vessels is a screw propeller. It has a simple design, and it is reliable and efficient. However, the conventional propeller has a number of disadvantages. Among them are the presence of cavitation and generation of the associated under-water noise. Another big disadvantage associated with a propeller is a shaft-sealing problem. This problem is especially serious for deep-water exploration submarines operating under high hydrostatic pressures, whereas the need in such submarines has become apparent during the ecological disaster a few years ago at one of the deep-water oil rigs in the Gulf of Mexico.

To overcome the above-mentioned problems associated with a propeller, one could use alternative aquatic propulsive systems, in particular those taking inspiration from nature and attempting to emulate undulating fish swimming modes by using natural or artificially simulated wave motion in different immersed structures.

One of the first investigations of this kind has been undertaken by Botman (1965) who demonstrated the feasibility of using a mechanically excited undulating plate to propel a model catamaran. He has demonstrated experimentally that this type of propulsion is viable and it has a number of advantages over a propeller, such as the absence of shaft-sealing problem, low underwater noise (due to the absence of cavitation), safe environment for 
swimmers, small idling drag and good thrust control. Paidoussis (1976) used a similar model catamaran with a submerged undulating plate. It is important to note that in both investigations mentioned above the authors tried to emulate the so-called 'anguilliform' fish swimming mode, which is a subcategory of the more general type of body and/or caudal fin locomotion (BCF) (Stakiotakis et al, 1999; Paidoussis, 2004; Colgate et al, 2004).

In more recent works, the anguilliform and other types of BCF locomotion have been subjected to numerous investigations and engineering imitations, using undulating plates, simple oscillating fins, or artificial waving structures made of linked fragments, each of them being actuated by a separate servo motor (Triantafyllou and Triantafyllou, 1995; Triantafyllou et al, 2000; Yamamoto et al, 1995; Sfakiotakis et al, 1999; Wolfgang et al, 1999; Guo et al. 2003; Guglielmini et al, 2003; Schouveiler et al, 2005; Terada et al, 2006; Heo et al, 2007). One should note, however, that practical applications of anguilliform and other types of BCF propulsion are limited to unmanned autonomous underwater vehicles (AUV), which can be used for research and surveillance operations. For manned vessels the anguilliform and BCF propulsion is unsuitable, as the main body of the vessel in this case would be subject to intensive vibrations in reaction to the propulsion, which would make onboard conditions unsustainable for the crew and passengers.

For the above reason, emulating of another type of fish locomotion, the so-called median and/or paired fin (MPF) locomotion seems to be more suitable for manned vessels. One of the subcategories of this locomotion, called 'rajiform', which seems to be most suitable, is used in nature by stingrays and skates (Stakiotakis et al, 1999). There are several published experimental works that try to emulate rajiform mode of swimming in order to propel marine vessels (see e.g. Takagi et al, 2006; Low, 2009). In all these works, the authors use artificially created waving fins made of linked elements actuated by separate servo motors, which results in rather complicated constructions of the propulsion systems. 
The idea of wave-like aquatic propulsion of manned marine vessels that is considered in this paper has been first published by the present author (Krylov, 1994). This idea is based on employing the 'rajiform' type locomotion which is implemented using a special type of localised (guided) flexural waves freely propagating along edges of wedge-like structures immersed in water, also known as 'wedge elastic waves' (see Fig. 1). Different wedge-like structures supporting this type of guided localised elastic waves can be attached to a body of a small ship or a submarine as keels or wings that are to be used for aquatic propulsion.

The above-mentioned wedge elastic waves propagating in contact with water have been first predicted and investigated theoretically by the present author (Krylov, 1994, 1998). The principle of using localised elastic waves as a source of aquatic propulsion is similar to that used in nature by stingrays. It is vitally important for the application of localised elastic waves for propulsion of marine vessels that, in spite of vibration of the fins, the main body of the craft remains undisturbed because the energy of localised waves is concentrated near the wings' tips (Krylov, 1994). This permits this method to be used for propulsion of manned marine vessels.

Comparing this method of propulsion with the methods developed in the other works emulating rajiform type locomotion using systems of actuators (Takagi et al, 2006; Low, 2009), one can conclude that the former is much simpler as it uses natural wave propagation and does not need numerous actuators to simulate the localised propulsive wave. Such a wave already exists in the immersed fin structure under consideration, and only one actuator is needed to excite the wave of desirable frequency and amplitude.

The advantages of this method, in comparison with traditional methods of propulsion, such as propellers and jets, are largely the same as those associated with BCF propulsion and mentioned by Botman (1965) and Paidoussis (1976). Namely, the absence of propeller shaftsealing problem, low underwater acoustic noise (due to the absence of cavitation), safe 
environment for swimmers, small idling drag, good thrust control, and the ability to propel vessels in muddy and weed-infested environments. The main envisaged applications of the proposed type of wave-like propulsion are small and medium sized manned deep-water exploration submarines, as well as autonomous underwater vehicles (AUV). Other possible applications can be for small and medium surface marine vessels, in particular for sailing boats (in times of low wind).

Wedge elastic waves that are to be used for this type of propulsion are very complex in nature. As a rule, they can be described only numerically, even for the simplest case of wedges in vacuum which was first considered back in the 1970's by Lagasse (1972) and Maradudin et al (1972) (see also the book of Biryukov et al (1995) and references therein). The degree of complexity is even higher for wedges in contact with water. However, for an important case of slender wedges the situation can be simplified in both cases by using the geometrical acoustics approximation. Using this approximation, one can solve the equations for bending vibrations of slender wedges of arbitrary shapes. As a result, one can obtain relatively simple and physically explicit solutions for localised waves propagating in wedges in contact with water (Krylov, 1994, 1998; Krylov et al, 2000; Shuvalov et al, 2000). Other known approaches to analysing wedge elastic waves in contact with water include WienerHopf techniques (Shanin et al, 2000) and finite element calculations (Hladky-Hennion et al, 1997). It should be noted that wedge elastic waves in contact with water that should be used for propulsion are waves propagating in the subsonic regime of wave propagation (in comparison with the speed of sound in water). As it is well known, such waves do not generate sound in the surrounding water. Adding to this the absence of cavitation normally associated with propellers, one could expect that the proposed wave-like propulsion should be much quieter than a propeller. 
For the purpose of aquatic propulsion of manned vessels, one can use wedge waves propagating in wedges of any shapes, including linear wedges. All of them provide localisation of the wave energy in the lateral direction. The most suitable, however, appear to be quadratic wedges, which local thickness is described by the function $h(x)=\varepsilon x^{2}$, where $x$ is the distance from the edge and $\varepsilon$ is a constant. In such wedges, all modes of guided flexural waves are dispersive, i.e. their phase velocities depend on frequency (Krylov et al, 2000). In the case of using these waves for aquatic propulsion, this would allow an operator of a vessel to change wedge wave velocity by changing frequency, which may be convenient for efficient start and acceleration of the vessel from rest. Another advantage of using quadratic or higher profile wedges is that they utilise a larger proportion of their surfaces for localised wave propagation in comparison with linear wedges, which again is beneficial for aquatic propulsion.

Although waves in quadratic wedges seem to be the most suitable for aquatic propulsion of manned vessels, it is rather difficult to use them in the initial experimental investigations due to difficulties in manufacturing of experimental quadratic or higher profile wedges. To avoid this problem on the initial stage it is convenient to use the earlier established similarity between guided wave propagation in quadratic wedges and in the systems comprising thin ridges embedded into an elastic half-space (Krylov, 1990b). The latter systems are in turn similar to thin rectangular plates with one long edge being free and another one being clamped. Therefore, for experimental purposes, one can use guided flexural waves propagating along free edges of the 'clamped-free' plates (Fig. 2), instead of quadratic wedges. One should keep in mind though that, in contrast to quadratic wedges, such plates do transmit vibrations to the main body of a vessel. Therefore, they can not be recommended for applications in real-life manned vessels. 
The first practical implementation and experimental testing of this type of aquatic propulsion have been carried out using a small model catamaran (Fig. 3) employing guided localised flexural waves propagating in a vertical clamped-free rubber plate (Krylov et al, 2007a,b). The test results have shown that the catamaran was propelled quite efficiently and could achieve the speed of $36 \mathrm{~cm} / \mathrm{s}$ (or about one body length per second), thus demonstrating that the idea of wave-like aquatic propulsion of manned craft by propagating flexural waves is viable.

The above investigation was followed by the design and experimental testing of a smallscale mono-hull model boat (Krylov et al, 2010) propelled by a localised flexural wave propagating along the free edge of a clamped-free rubber plate forming the boat's keel (see Fig. 4). Experimental investigations have been carried out in a water tank (Fig. 5) and in open water conditions. The open-water tests included measurements of the boat speed as well as measurements of drag, thrust and propulsion efficiency. The propulsive plate was driven at the front edge by the exciter bar (Figs. 4 and 5). The exciter bar, which was driven by a servo motor, has been designed to allow maximum angle of $30^{\circ}$ to be achieved either side of the centre line. With the exciter bar length used this gave a maximum amplitude of flexural wave displacement of $33 \mathrm{~mm}$. To actuate the exciter bar of the propulsion system under consideration a commercial programmable servo motor was used. The measured results for steady state craft speed as a function of frequency and amplitude (see Fig. 6) have demonstrated the viability and efficiency of this type of propulsion (the maximum swimming speed was about $32 \mathrm{~cm} / \mathrm{s}$ ). The results for generated thrust (see Fig. 7) have been calculated from the measured values of steady-state swimming speed (Fig. 6) using the experimentally measured dependence of the craft's drag as a function of swimming speed.

It should be noted that the idea and method of wave-like aquatic propulsion described above have been subsequently explored for an underwater model of a 'robotic fish' (Liu et al, 
2010). The propulsive system used by the authors was similar to the one that was used earlier to propel a model catamaran (Krylov et al, 2007a,b). Experimental measurements of generated thrust have shown its positive value. However, the numerical calculations carried out by the authors using the computational fluid dynamics (CFD) method predicted a negative value of thrust, which was in contradiction with the experiments.

In light of the above-mentioned successful experimental demonstrations of feasibility of the wave-like aquatic propulsion using guided flexural waves, it is important to achieve better understanding of the processes of generating hydrodynamic thrust by freely propagating flexural waves. Solution to this problem would allow the developers to determine optimal parameters of propagating waves and of the geometry of propulsive wedges and plates providing maximum efficiency of the propulsion.

The aim of the present paper is to report the results of the initial theoretical research into the role of nonlinear distortion of propagating flexural waves in generating thrust that could be applied to small marine craft experimentally tested in the papers by Krylov et al (2007a,b; 2010). The main motivation for this investigation was the fact that the amplitudes of flexural waves used for propulsion of the model craft were large enough, so that nonlinear effects were expected to be important.

It was natural to use the well-known Lighthill's theory of fish locomotion (Lighthill, 1960; 1970) as the basis for analysing the role of nonlinear effects in generating thrust. It should be noted that Lighthill's theory in its standard form predicts zero thrust at all frequencies when applied to flexural waves of constant amplitude. Note in this connection that the localised flexural waves used for propulsion in the papers by Krylov et al (2007a,b; 2010) were excited from the front edges of the propulsive plates via mechanical arms, which means that the amplitudes of these waves were maximal at the front edges of the propulsive plates and were decreasing (or at best were kept constant) over the length of the plates 
towards the rear edges. According to Lighthill's formula (Lighthill, 1960), this results in zero thrust, which is in contradiction with the above-mentioned experiments, in particular with the measured nonzero values of generated thrust (see Fig. 7). One should note in this connection that Lighthill's theory of fish locomotion usually assumes that the amplitudes of propulsive waves created by fish body motion grow from zero on the front (at fish heads) to their maximum values at the tails. This is consistent with fish body motion in nature, but is not compatible with the behaviour of the propagating localised flexural waves employed for propulsion in the model vessels used in the experiments of Krylov et al (2007a,b; 2010).

It is suggested in the present paper that nonlinear distortion of localised flexural waves in the process of their propagation may play an important role in generating thrust in real experimental marine craft. Indeed, the Mach numbers of propagating flexural waves used for propulsion in the above-mentioned experimental works were as large as about two (Krylov et al, 2007a), which makes the above suggestion quite realistic. This hypothesis is explored in the present work by adding nonlinear harmonics of propulsive flexural waves, that are growing with the distance of propagation due to elastic nonlinearity, to the Lighthill's formula for generated thrust. For simplicity, only the lowest (third) harmonic of the localised flexural waves is used, similarly to the earlier work by Krylov et al (1992). Also for simplicity, the effect of wave dispersion on generation of the nonlinear harmonic is neglected, assuming that the plate-like structures used in the experiments are short enough in the direction of wave propagation. The sum of the initial time-harmonic wave and its third nonlinear harmonic having the amplitude linearly increasing with the distance of propagation is then substituted into Lighthill's formula to derive the analytical expression for the averaged thrust.

The derived analytical expression for the averaged thrust shows that, due to the effect of the third harmonic, the thrust calculated according to Lighthill's formula is no longer zero, thereby demonstrating that nonlinear distortion of the propulsive flexural waves may be 
important for accurate description of wave-like aquatic propulsion of small marine craft employing freely propagating flexural waves. Using the derived expression, the initial numerical estimates of the generated thrust are carried out for the parameters of the experimental vessels and of the propulsive flexural waves used in the experiments.

\section{Theoretical background}

\subsection{Lighthill's approach to the theory of wave-like aquatic propulsion}

One of the first theoretical papers on wave-like aquatic propulsion in application to fish locomotion has been published by Lighthill (1960) who developed a three-dimensional elongated body theory (EBT) (see also the paper of Cheng et al (1994)). Soon after Lighthill's publication, a two-dimensional theory applied to an infinite 'waving plate' has been published by $\mathrm{Wu}$ (1961). Since a three-dimensional approach seems to be more appropriate for description of the experimental propulsive systems used in the papers of Krylov et al (2007a,b; 2010), we will use Lighthill's approach in further consideration.

In his analysis of the problem, Lighthill considered a slender fish that remains stationary in a steady flow of water with the velocity $U$ in the $x$-direction. It is assumed that when the fish is motionless, or 'stretched straight', there is no normal force acting upon the cross section. It was also assumed that the motion of the fish at any particular cross section can be modelled as a displacement $h$ in the perpendicular direction (z-direction), which is a function of $x$ and $t$. This displacement causes the velocity of the fluid flowing past the cross section to change from the initial value of $U$ to a new value, $V$, which is also a function of distance and time: 


$$
V(x, t)=\frac{\partial h}{\partial t}+U \frac{\partial h}{\partial x}
$$

The mean mechanical work over a long time $\bar{W}$ done by the fish by making displacements $h(x, t)$ can be expressed in the form (Lighthill, 1960):

$$
\bar{W}=\rho U\left[\overline{\frac{\partial h}{\partial t} V A(x)}\right]_{0}^{L},
$$

where $A(x)$ is the area of the circumscribing circle of the ellipse-shaped cross section of the fish. Lighthill then assumes that the function $A(x)$ and/or $h(x, t)$ has a zero value for $x=0$ (at fish head). He also assumes that $h(x, t)$ grows towards the tail to reach its maximum there (at $x=L$ ), which is a good approximation for real fish body motion in nature. In these cases Eqn (2) reduces to

$$
\bar{W}=\rho U\left[\overline{\frac{\partial h}{\partial t} V A(x)}\right]_{X=L},
$$

which results in the following expression for the total thrust produced (Lighthill, 1960):

$$
\bar{P}=\frac{1}{2} \rho A(L)\left\{\overline{\left(\frac{\partial h}{\partial t}\right)^{2}}-U^{2} \overline{\left(\frac{\partial h}{\partial x}\right)^{2}}\right\}_{x=L} .
$$


Let us now apply the above expressions to the above-described experimental marine craft investigated in the papers by Krylov et al (2007a,b; 2010), keeping in mind that localised flexural waves used for propulsion in these papers were excited from the front edges of the propulsive plates via mechanical arms, which means that, contrary to Lighthill's assumption of $h(x, t)=0$ at $x=0$, the amplitudes of these waves were maximal at the fronts of the propulsive plates (at $x=0$ ) and were decreasing (or at best were kept constant) over the length of the plates towards the rear edges. Also, instead of $A(x)=0$ at $x=0$, there is $A(x)=$ $A=$ const $\neq 0$.

Let us assume that the flexural waves that were generated in the experiments of the papers by Krylov et al (2007a,b; 2010) are time-harmonic and have a constant amplitude $H$ along the length of the flexible plate (fin):

$$
h(x, t)=H \cos (\omega t-k x) .
$$

Here $k=\omega / c$ is the wavenumber of the localised flexural wave, where $\omega$ is the circular frequency, and $c$ is the velocity of the localised wave propagation along the propulsive plate. It must be noted that in the case of localised flexural waves used in the experiments the wave amplitudes were not constant at different points along the perpendicular direction (y-axis). However, for simplicity, it is assumed in Eqn (5) that the amplitudes are constant everywhere over the plate.

As it follows from Eqn (5), the Lighthill's assumption of $h(x, t)=0$ at $x=0$ is no longer applicable, and in order to calculate the mean mechanical work $W$ one should use Eqn (2) instead of Eqn (3). Similarly, instead of using Eqn (4) to calculate thrust, one should use the full expression 


$$
\bar{P}=\frac{1}{2} \rho A\left\{\overline{\left(\frac{\partial h}{\partial t}\right)^{2}}-U^{2} \overline{\left(\frac{\partial h}{\partial x}\right)^{2}}\right\}_{0}^{L}
$$

Here $A=\pi\left(d^{2} / 4\right)$, where $d$ is the width of the propulsive plate.

Substituting Eqn (5) into Eqn (6), one can see that the resulting long-time average thrust is zero. This means that, according to Lighthill's theory, there is no thrust produced by a propulsive wave of constant amplitude described by Eqn (5). This contradicts the experimental results of Krylov et al (2007a,b; 2010) showing that there is a significant amount of thrust generated (see Fig. 7). Therefore, one can conclude that the abovementioned theoretical analysis must be neglecting some mechanisms that are present in real experiments and are responsible for generation of non-zero thrust.

\subsection{Some other theoretical approaches}

It should be noted that the numerical calculations using CFD (Shirgaonkar et al, 2008) do predict a non-zero thrust generated by linear harmonic waves of constant amplitude propagating along a ribbon-fin system similar to the propulsive plates used in the papers of Krylov et al (2007a,b; 2010). Moreover, the general behaviour of the predicted thrust, in particular its growth with frequency, is in line with the experimental observations of Krylov et al (2007a,b; 2010). The earlier developed analytical approach to a similar propulsive system (Lighthill et al., 1990) also predicts a non-zero thrust, but the results are significantly underestimated in comparison with the paper of Shirgaonkar et al. (2008).

As was mentioned above, the numerical calculations in the paper of Liu et al (2010) have predicted the negative value of thrust for a similar system propelling a 'robotic fish'. In their calculations, the authors considered the propulsive wave with the amplitude decaying with 
propagation distance. Therefore, this result is in agreement with Lighthill's formula for this case, but it is in contradiction with the experimental observations of the same authors demonstrating a positive thrust for this model.

It is suggested in the present paper that one of the 'missing' mechanisms responsible for generation of non-zero thrust, in addition to those that can be captured by CFD (Shirgaonkar et al, 2008), may be the nonlinear waveform distortion (Vinogradova et al, 1979; Whitham, 1974) of the propulsive flexural waves during their propagation from the front to the rear edge of the plate. A detailed exploration of this idea is presented in the next section.

\section{Accounting for generation of nonlinear harmonics}

In this section, we explore the hypothesis that one of the mechanisms contributing to a positive thrust observed in the experiments described in the papers by Krylov et al. (2007a,b; 2010) may be the nonlinear distortion of the waveforms of the propulsive flexural waves during their propagation. Using a spectral interpretation, this nonlinear distortion can be described as a result of generation of higher order harmonic waves propagating along the length of the propulsive plate. This hypothesis is backed up by the experimental data from the paper by Krylov et al (2010), as the results for the lowest wave amplitude tested (15 mm displacement) were practically zero, and only at the larger amplitudes there was a significant thrust achieved (see Fig. 7).

In the earlier published theoretical paper on generation of nonlinear harmonics in wedge elastic waves (Krylov et al, 1992), it was shown that in the case of anti-symmetric localised waves, which is also the case for the propulsive waves used in the above-mentioned experiments (Krylov et al, 2007a,b; 2010), the lowest order of nonlinearity is the third order, 
as the quadratic term vanishes because of the symmetry of the problem. Therefore, the description of the nonlinear distortion of the time-harmonic propulsive waves for the problem under consideration can be limited to the accounting for the third nonlinear harmonic only, for simplicity.

In light of the above, let us consider the addition of a nonlinear third harmonic to the Lighthill's formula, Eqn (6). Based on the results of Krylov et al (1992), we will assume that the amplitude of the generated third harmonic is proportional to $H^{3}$, and it grows with distance $x$ linearly, starting from zero at the front edge of the propulsive plate. The addition of the third harmonic thus changes the expression (5) for $h(x, t)$, which now takes the form:

$$
h(x, t)=H \cos (\omega t-k x)+F_{n l}(H) x \cos (3 \omega t-3 k x-\psi) .
$$

Here $F_{n l}(H)$, which is proportional to $H^{3}$, is a non-dimensional function describing the effect of nonlinearity, and $\psi$ is the initial phase for the nonlinear term. The function $F_{n l}(H)$ also depends on the nonlinear elastic moduli of the material of the propulsive plate, which constitutes 'elastic nonlinearity'. In what follows we will assume that the material of the propulsive plate is rubber, as it was in the experiments (Krylov et al, 2007a,b; 2010).

In the expression (7), it is assumed that $H=$ const, i.e. that the amplitude of the first (main) harmonic does not change with the distance as a result of nonlinear distortion. This initial rather rough approximation, which can be called the 'approximation of a given field' (Vinogradova et al, 1979), will be considered here first. Later on, we will take the change of amplitude of the first harmonics into account using energy conservation law.

Substituting Eqn (7) into Eqn (6) and doing the required operations, we obtain that the generated thrust is no longer zero, and it is defined by the following expression: 


$$
\bar{P}=\frac{9}{4} \rho A F_{n l}^{2}(H) L^{2} \omega^{2}\left(1-\frac{U^{2}}{c^{2}}\right)
$$

It follows from Eqn (8) that thrust is generated due to the nonlinearity if the expression in brackets is positive, i.e. when $c>U$, which is the usual condition for the velocity $c$ of an elastic wave propagating along the plate to be larger than the velocity of swimming $U$. Although the expression (8) for the thrust is meaningful, the correctness of the assumption $H$ $=$ const in application to this problem does not look very convincing.

For that reason, we now consider a more refined approach taking into account the change in the amplitude of the first harmonic with the distance of propagation because of the nonlinear generation of the third harmonic. This can be done using energy conservation law. In the approximation of only two interacting harmonics, the first and the third, this law takes the form

$$
\omega^{2} H^{2}=\omega^{2} H^{2}(x)+(3 \omega)^{2} H_{3}^{2}(x)=\omega^{2} H^{2}(x)+9 \omega^{2} F_{n l}^{2}(H) x^{2},
$$

where $H$ now denotes the initial amplitude of the first harmonics (at $x=0$ ), and $H(x)$ represents its changing value at $x>0$. It follows from Eqn (9) that the changing amplitude $H(x)$ can be expressed as

$$
H(x)=\sqrt{H^{2}-9 F_{n l}^{2}(H) x^{2}} .
$$

We now expand the expression in the right-hand side of Eqn (10) into the Taylor series, retaining terms up to the fourth order in $F_{n l}$. This gives the following approximate expression for $H(x)$ : 


$$
H(x)=H-\frac{9 F_{n l}^{2}(H) x^{2}}{2 H}+\frac{81 F_{n l}^{4}(H) x^{4}}{8 H^{3}} .
$$

Let us now replace Eqn (7) by the more precise expression taking into account the changing amplitude of the first harmonic $H(x)$ according to Eqn (11),

$$
h(x, t)=\left(H-\frac{9 F_{n l}^{2}(H) x^{2}}{2 H}+\frac{81 F_{n l}^{4}(H) x^{4}}{8 H^{3}}\right) \cos (\omega t-k x)+F_{n l}(H) x \cos (3 \omega t-3 k x-\psi),
$$

and substitute it into Eqn (6) for generated thrust, keeping terms up to the fourth power of $F_{n l}(H)$. After rather bulky derivations, it can be shown that all terms of the second order in $F_{n l}(H)$ cancel each other, and the resulting expression for generated thrust takes the form

$$
\bar{P}=\frac{1}{2} \rho A \frac{81}{4} \frac{F_{n l}^{4}(H) \omega^{2} L^{4}}{H^{2}}\left[1-\frac{U^{2}}{c^{2}}\left(1+\frac{2}{k^{2} L^{2}}\right)\right]
$$

where $k=\omega / c$ is the wavenumber of the propagating flexural wave. This means that the initial 'approximation of a given field', Eqn (7), is insufficient, and the expression for generated thrust, Eqn (8), following from that approximation is incorrect as it does not take into account some terms of the second order in $F_{n l}(H)$ that appear due to the change of amplitude of the first harmonic with the propagation distance, which results in the mutual cancellation of all terms of the same (second) order in $F_{n l}(H)$, and thus in zero thrust generated in this order of nonlinearity. Using a more refined formula for $h(x, t)$, Eqn (12), 
and keeping all the terms of the fourth order versus $F_{n l}(H)$ in further derivations results in the expression for generated thrust, Eqn (13), that is proportional to the fourth power of $F_{n l}(H)$.

It is convenient to simplify Eqn (13) using a typical relationship between the parameters of the problem. Usually, $L \approx 2 \lambda=4 \pi / k$. Therefore, $k^{2} L^{2} \approx 16 \pi^{2} \approx 158$ and $2 /\left(k^{2} L^{2}\right) \approx 0.013$, which is much less than 1 . Thus, the second term in round brackets can be neglected. This results in a simplified expression for generated thrust:

$$
\bar{P}=\frac{1}{2} \rho A \frac{81}{4} \frac{F_{n l}^{4}(H) \omega^{2} L^{4}}{H^{2}}\left(1-\frac{U^{2}}{c^{2}}\right) .
$$

Let us now specify the nonlinear function $F_{n l}(H)$. The easiest way to proceed is to use the results of the work by Krylov et al (1992), where this function has been calculated for wedge elastic waves in linear wedges, which are localised non-dispersive flexural modes propagating along wedge tips (Fig. 1). Although linear elastic wedges are not exactly the structures that have been used in the experiments of Krylov et al (2007a,b; 2010), they are interesting for their own sake and they also can be used for rough estimates of the experimental situation. According to Krylov et al (1992), the function $F_{n l}(H)$ for linear elastic wedges in vacuum takes the form

$$
F_{n l}(H)=\frac{1}{4} \frac{P}{Q} \frac{b}{a} \frac{\theta^{2}}{n^{2}}(k H)^{3} .
$$

Here $P$ and $Q$ are dimensionless parameters depending on modal shapes of wedge modes, $a=E / 12\left(1-\sigma^{2}\right)=\rho_{s} c_{p}^{2} / 12$ is a non-specified parameter, where $E$ is the Young's modulus, $\sigma$ is the Poisson's ratio, $\rho_{s}$ is the mass density of the wedge material, and $c_{p}$ is the velocity of 
plate compression waves for the wedge material, $\theta$ is the wedge angle, and $k=\omega / c$ is the wavenumber of a wedge mode characterised by the number $n=1,2,3, \ldots$, where $c$ is the velocity of wedge mode of number $n$ (for shortness, $k$ and $c$ are written without index $n$ ), and the value of $\psi$ in Eqn (12) should be taken as $-\pi / 2$. The parameter $b=0.4 f\left(c_{t} / c_{l}\right)^{6}$ describes the nonlinear properties of the structure. Here $c_{t}$ and $c_{l}$ are the velocities of shear and longitudinal elastic waves in the wedge material, and $f$ is the relevant 4th order elastic module describing cubic nonlinearity.

For linear elastic wedges immersed in water, we will calculate $F_{n l}(H)$ using the same Eqn (15), but with the wavenumbers $k=\omega / c$ being replaced by those for wedge waves in water. This means that instead of wedge wave velocities $c$ for wedges in vacuum (Krylov, 1989; 1990a),

$$
c=\frac{c_{p}}{\sqrt{3}} \theta n,
$$

we will use the expression for wedge wave velocities for wedges in water (Krylov, 1998),

$$
c=c_{t} A_{0}^{-5 / 2} D^{-3 / 2}(\pi n)^{3 / 2} \theta^{3 / 2},
$$

where

$$
A_{0}=6^{1 / 5}\left(\frac{\rho}{\rho_{S}}\right)^{1 / 5}[2(1-\sigma)]^{1 / 5}
$$

and $D=2.102$. 


\section{Numerical calculations and discussion}

Numerical calculations have been carried out to determine the thrust generated by the first order $(n=1)$ localised wedge mode for a given swimming speed $U$ using formulas (14), (15) and (17), (18) for the parameters of the problem shown in Table 1. Because of the lack of reliable information about the forth order elastic moduli of rubber, we used a typical value of the relation between the forth and second order moduli, assuming that $f / E \approx 10$. For the fraction $P / Q$, we took the estimated value of 2 , which was based on the numerical calculations of the paper by Krylov et al (1992). For convenience of comparison with the experiments (Krylov et al, 2010), the thrust was calculated in gramms, rather than in Newtons. We remind the reader that the relationship between the same forces $F$ expressed in Newtons $(\mathrm{N})$ and in gramms $(\mathrm{g})$ is

$$
F_{(g)}=F_{(N)} \frac{g}{1000},
$$

where $g=9.81 \mathrm{~m} / \mathrm{s}^{2}$ is gravity acceleration.

Before discussing the results for generated thrust, it is instructive to visualise the nonlinear waveform distortion of the propagating localised flexural wave calculated according to Eqns (12) and (15). The results are presented in Fig. 8; the waveforms are shown for the initial harmonic wave at frequency $f=4.5 \mathrm{~Hz}$ and amplitude $H=28 \mathrm{~mm}$ (at $x=0$ ) and for the nonlinearly distorted wave after its propagation until the point $x=0.17 \mathrm{~m}$.

The results of the calculations of thrust are shown in Fig. 9 in the frequency range $2-4.8$ $\mathrm{Hz}$ for the flexural wave amplitude $H=28 \mathrm{~mm}$. For comparison, the experimental results of Krylov et al (2010) are also shown in Fig. 9 by boxes (see also Fig. 7 for experimental data). 
The results of the calculations of thrust for the flexural wave amplitude $H=22 \mathrm{~mm}$ are shown in Fig. 10 in the same frequency range. Also, the corresponding experimental results of Krylov et al (2010) are shown in Fig. 10 by boxes.

As can be seen from Figs. 9 and 10, the nonlinear mechanism gives rather large contributions to thrust at higher frequencies, but it significantly underestimates the experimental values of thrust at lower frequencies. This means that the nonlinear mechanism of thrust generation is important at higher frequencies. However, in the current stage of the theory, its contribution to thrust grows very rapidly with frequency, which does not agree well with the experimental values. One of the possible reasons for that could be the fact that elastic wedges of linear profile have been used in this paper for modelling the real plate-like propulsive structures used in the experiments. Wedge elastic waves propagating in wedges of linear profile have no dispersion, whereas localised flexural waves propagating in clampedfree plates used in the experimental propulsive systems are dispersive. This could have a substantial effect on limitation of the nonlinear generation of the third harmonic in the experimental plate-like propulsive structures. Further theoretical and experimental research would be required in this direction.

\section{Conclusions}

The results of the initial research into the effect of elastic nonlinearity on the theory of aquatic propulsion by propagating flexural waves presented in this paper demonstrate that the nonlinear distortion of the propagating flexural waves may be important for generating a positive thrust at higher frequencies. 
The comparison between the theoretical calculations of generated thrust and the experimental measurements at higher frequencies, where the contribution of nonlinear distortion is essential, shows that the theory predicts a too rapid increase of thrust with frequency. One of the possible reasons for that could be the modelling of the real plate-like propulsive structures used in the experiments by elastic wedges of linear profile that cause no dispersion for propagating localised flexural waves and thus facilitate the development of nonlinear effects during wave propagation.

Further theoretical and experimental research in this direction is needed. In particular, it would be important to acquire experimental evidence of the nonlinear distortion of localised flexural waves propagating in immersed propulsive wedges and plates.

\section{References}

Biryukov, S.V., Gulyaev, Yu. V., Krylov, V.V., Plessky, V.P., 1995. Surface Acoustic Waves in Inhomogeneous Media. Springer, Berlin.

Botman, M., 1965. Propulsion by undulating plates. Journal of Aircraft 2, 456-462.

Cheng, J.Y., Blikhan, R., 1994. Note on the calculation of propeller efficiency using elongated body theory. Journal of Experimental Biology 192, 169-177.

Colgate, J.E., Lynch, K.M., 2004. Mechanics and control of swimming: a review. IEEE Journal of Oceanic Engineering 29(3), 660-673.

Guglielmini, L., Blondeaux, P., Vittori, G., 2003. A simple model of propulsive oscillating foils. Ocean Engineering 31, 883-899.

Guo, S., Fukuda, T., Asaka, K., 2003. A new type of fish-like underwater microrobot. IEEE/ASME Transactions on Mechatronics 8, 136-141. 
Heo, S., Wiguna, T., Park, H.C., Goo, N.S., 2007. Effect of an artificial caudal fin on the performance of a biomimetic fish robot propelled by piezoelectric actuators. Journal of Bionic Engineering 4, 151-158.

Hladky-Hennion, A.-C., Langlet, P., De Billy, M., 1997. Finite element analysis of the propagation of acoustic waves along waveguides immersed in water. Journal of Sound and Vibration 200, 519-530.

Krylov, V.V., 1989. Conditions for validity of the geometrical-acoustics approximation in application to waves in an acute-angle solid wedge. Soviet Physics - Acoustics 35(2), 176-180.

Krylov, V.V., 1990a. Geometrical-acoustics approach to the description of localized vibrational modes of an elastic solid wedge. Soviet Physics - Technical Physics 35(2), 137-140.

Krylov, V.V., 1990b. Localized acoustic modes of a quadratic solid wedge. Moscow University Physics Bulletin 45 (6), 65-69.

Krylov, V.V., 1994. Propagation of wedge acoustic waves along wedges embedded in water. Proceedings IEEE Ultrasonics Symposium, Cannes, France, pp. 793-796.

Krylov, V.V., 1998. On the velocities of localized vibration modes in immersed solid wedges. Journal of the Acoustical Society of America 103, 767-770.

Krylov, V.V., Parker, D.F., 1992. Harmonic generation and parametric mixing in wedge acoustic waves. Wave Motion 15(2), 185-200.

Krylov, V.V., Shuvalov, A.L., 2000. Propagation of localised flexural vibrations along plate edges described by a power law. Proceedings of the Institute of Acoustics 22(2), 263270. 
Krylov, V.V., Pritchard, G.V., 2007a. Experimental investigation of the aquatic propulsion caused by localised flexural wave propagation in immersed wedges and plates. Applied Acoustics 68(1), 97-113.

Krylov, V.V.,. Pritchard, G.V., 2007b. Experimental confirmation of the propulsion of marine vessels employing guided flexural waves in attached elastic fins. Journal of Fluids and Structures 23, 297-307.

Krylov, V.V., Porteous, E., 2010. Wave-like aquatic propulsion of mono-hull marine vessels. Ocean Engineering 37, 378-386.

Lagasse, P.E., 1972. Analysis of a dispersionfree guide for elastic waves. Electronics Letters, 8(15), 372-373.

Lighthill, M.J., 1960. Note on the swimming of slender fish. Journal of Fluid Mechanics 9, 305-317.

Lighthill, M.J., 1970. Aquatic animal propulsion of high hydrodynamic efficiency. Journal of Fluid Mechanics 44, 265-301.

Lighthill, J., Blake, R., 1990. Biofluiddynamics of balistiform and gymnotiform locomotion. Part 1. Biological background, and analysis by elongated-body theory, Journal of Fluid Mechanics 212, 183-207.

Liu, F., Yang, C.J., Lee, K.M., 2010. Hydrodynamic modeling of an undulating fin for robotic fish design, Proceedings of the 2010 IEEE/ASME International Conference on Advanced Intelligent Mechatronics, Montréal, Canada, July 6-9, 2010, pp. 55- 60.

Low, K.H., 2009. Modelling and parametric study of modular undulating fin rays for fish robots. Mechanism and Machine Theory 44, 615-632.

Maradudin, A.A., Wallis, R.F., Mills, D.L., Ballard, R.L., 1972. Vibrational edge modes in finite crystals. Physical Review B, 6(4), 1106-1111.

Paidoussis, M.P., 1976. Hydroelastic ichthyoid propulsion. Journal of Hydronautics 10, 30-32. 
Paidoussis, M.P., 2004. Fluid-Structure Interactions: Slender Structures and Axial Flow, Vol. 2, Elsevier, Amsterdam. Section 8.10.

Schouveiler, L., Hover, F.S., Triantafyllou, M.S., 2005. Performance of flapping foil propulsion. Journal of Fluids and Structures 20, 949-959.

Sfakiotakis, M., Lane, D.M., Davies, J.B.C., 1999. Review of fish swimming modes for aquatic locomotion. IEEE Journal of Oceanic Engineering 24, 237-252.

Shanin, A.V., Krylov, V.V., 2000. An approximate theory for waves in a slender elastic wedge immersed in liquid. Proceedings of the Royal Society (London) A 456, 21792196.

Shirgaonkar, A.A., Curet, O.M., Patankar, N.A., MacIver, M.A., 2008. The hydrodynamics of ribbon-fin propulsion during impulsive motion. The Journal of Experimental Biology 211, 3490-3503.

Shuvalov, A.L., Krylov, V.V., 2000. Geometrical-acoustics consideration of the flexural modes in immersed anisotropic wedges. Journal of Sound and Vibration 237(3), 427434.

Takagi, K., Yamamura, M., Luo, Z.W., Onishi, M., Hirano, S., Asaka, K., Hayakawa, Y., 2006. Development of a rajiform swimming robot using ionic polymer artificial muscles. Proceedings 2006 IEEE/RSJ International Conference on Intelligent Robots and Systems. Beijing, China, October 9 - 15, 2006, pp. 1861 - 1866.

Terada, Y., Yamamoto, I., 2006. Robotic fish and its application. Proceedings 2006 IEEE Conference ISIE. Montreal, Canada, July 9-12, 2006, pp 3062-3065.

Triantafyllou, M.S., Triantafyllou, G.S., 1995. An efficient swimming machine. Scientific American 272, 64-70.

Triantafyllou, M.S., Triantafyllou, G.S., Yue, D.K.P., 2000. Hydrodynamics of fishlike swimming. Annual Review of Fluid Mechanics, 32, 33-53. 
Vinogradova, M.B., Rudenko, O.V., Sukhorukov, A.P., 1979. Wave Theory. Nauka, Moscow (in Russian).

Whitham, G.B., 1974. Linear and Nonlinear Waves. John Wiley and Sons, New York.

Wolfgang, M.J., Yue, D.K.P., Triantafyllou, M.S., 1999. Visualisation of complex near-body transport processes in flexible-body propulsion. Journal of Flow Visualisation 2, 143151.

Wu, T.Y., 1961. Swimming of a waving plate. Journal of Fluid Mechanics 10, 321-344.

Yamamoto, I., Terada, Y., Nagamatu, T., Imaizumi, Y., 1995. Propulsion system with flexible/rigid oscillating fin. IEEE Journal of Oceanic Engineering 20, 23-30. 


\section{Figures}

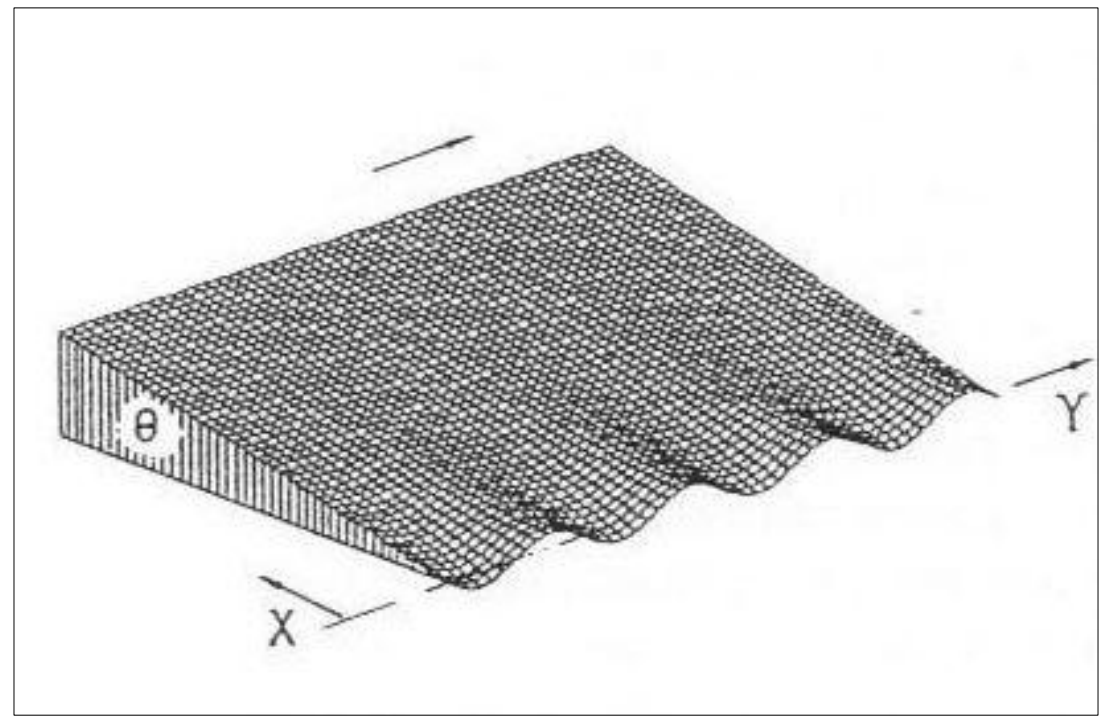

Fig. 1 Propagation of localised elastic waves along the tip of a linear wedge (Krylov, 1994). 


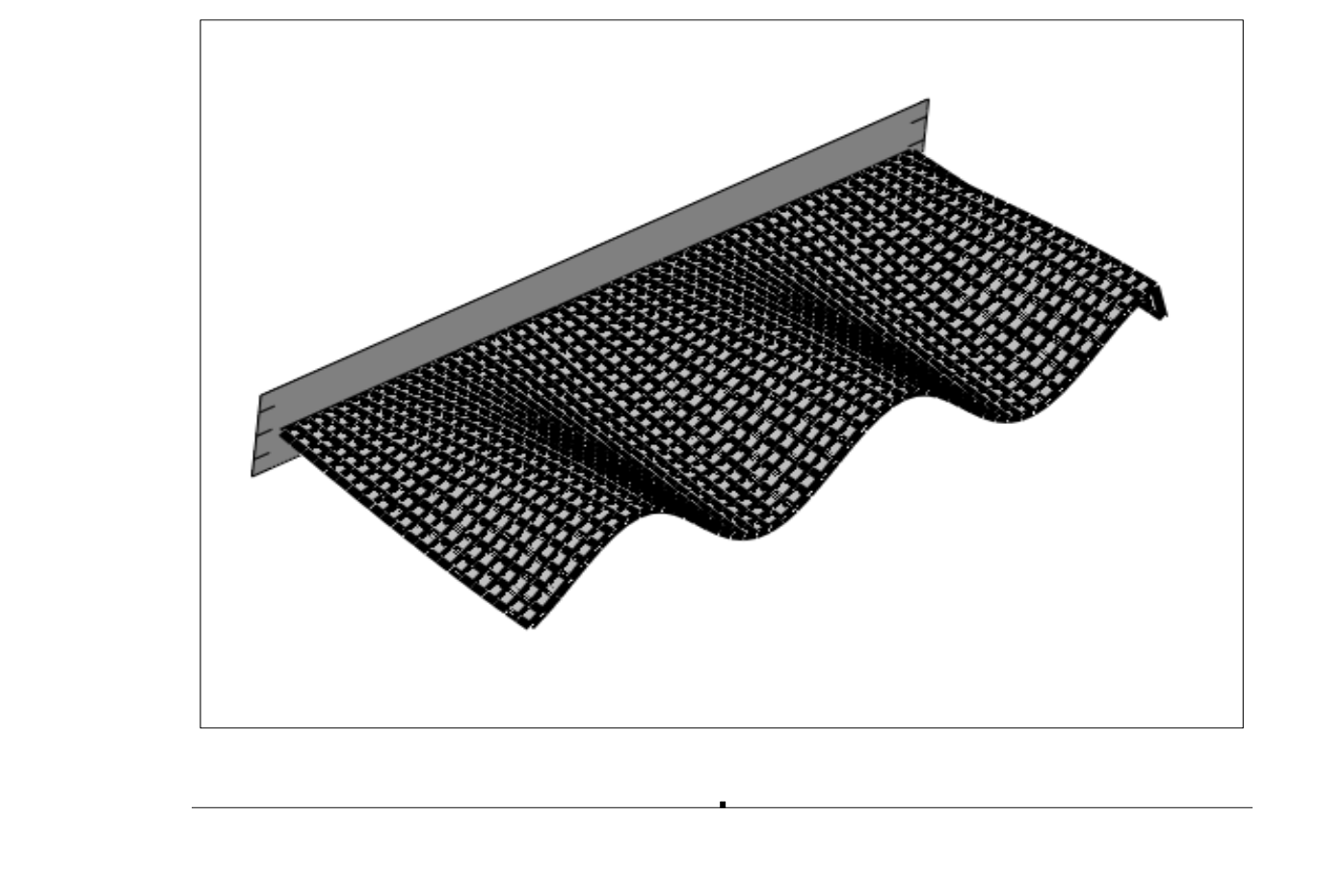

Fig. 2 Guided flexural waves propagating along the free edge of a clamped-free

plate. (a)

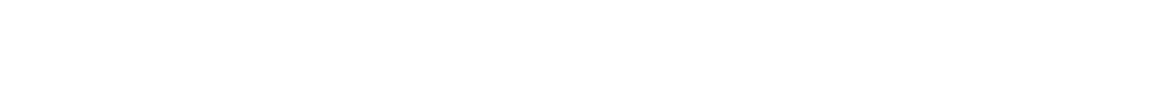

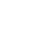

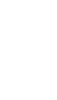

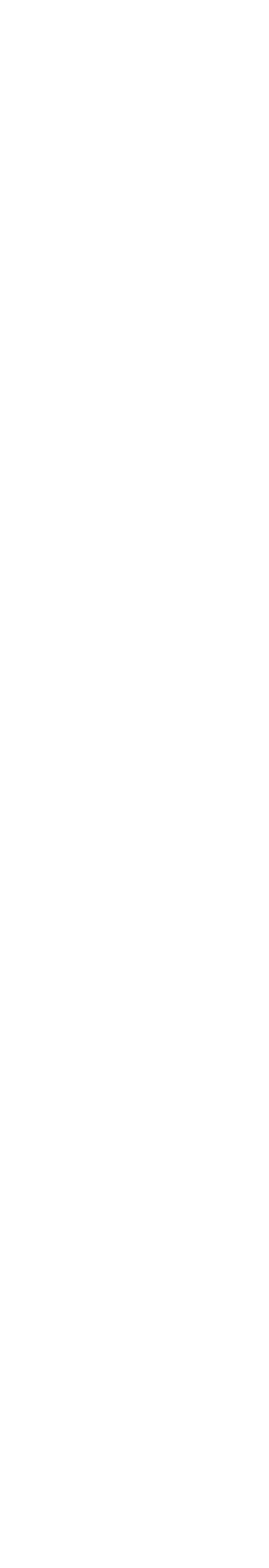

.

.

.

.

列

(1)

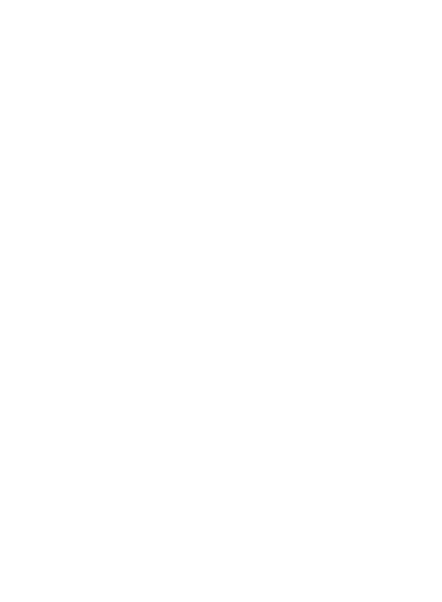




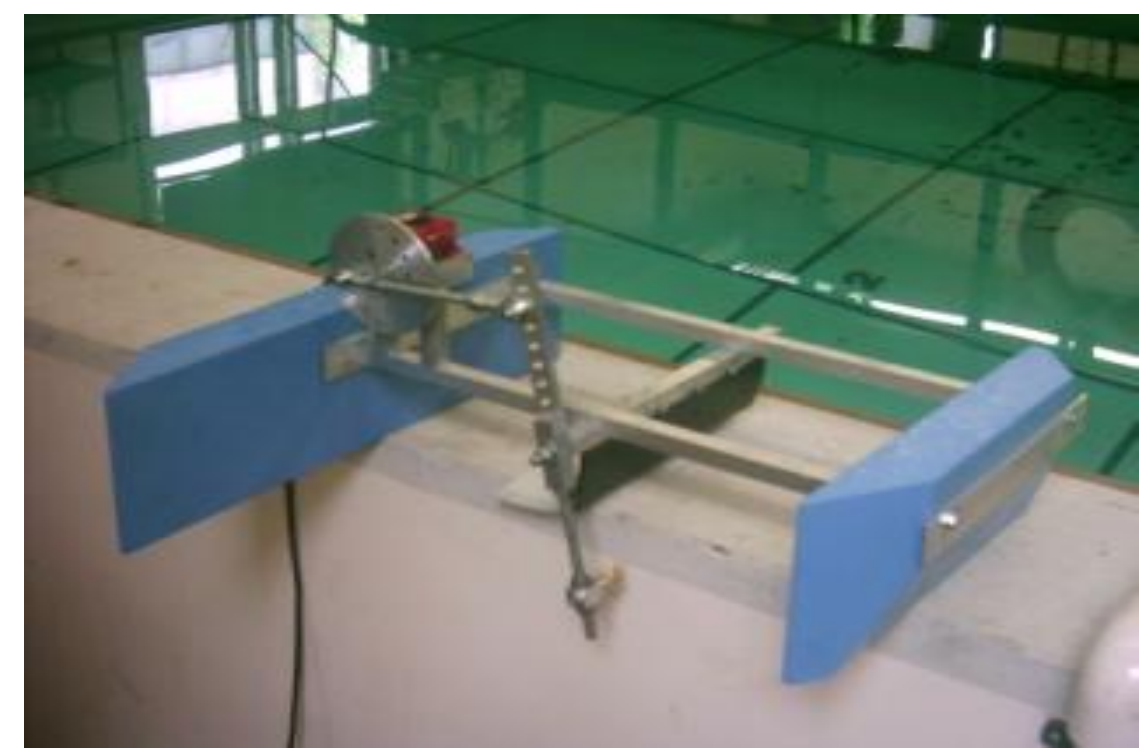

Fig. 3 View of the model catamaran with a propulsive rubber plate before testing in the experimental pool (Krylov et al, 2007a,b). 


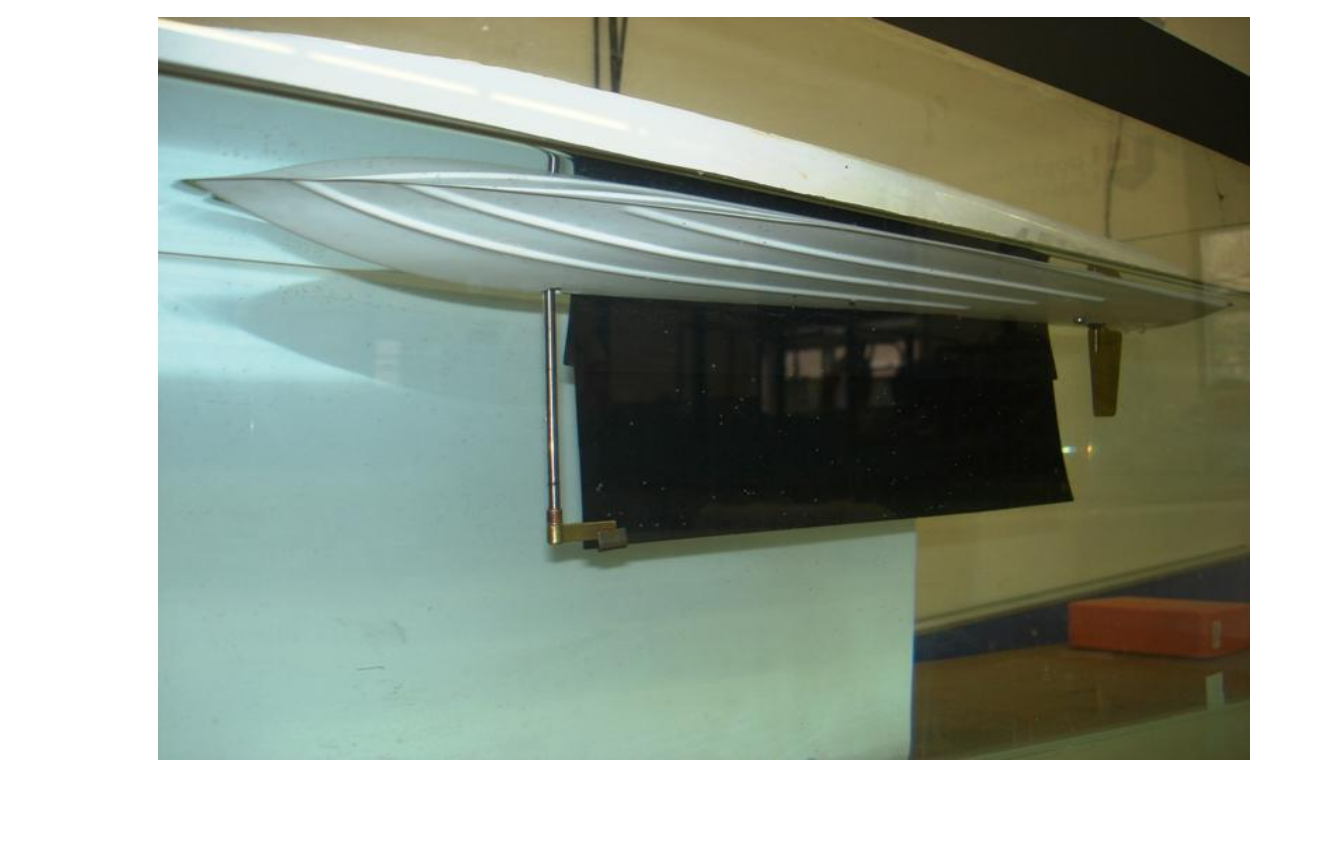

Fig. 4 Underwater view of the mono-hull model boat with the assembled propulsive rubber plate (Krylov et al., 2010).

\title{
Figure
}

Fig. 4 Underwater view of the mono-hull model boat with the assembled

\author{
propulsive rubber plate (Krylov et al., 2010).
}
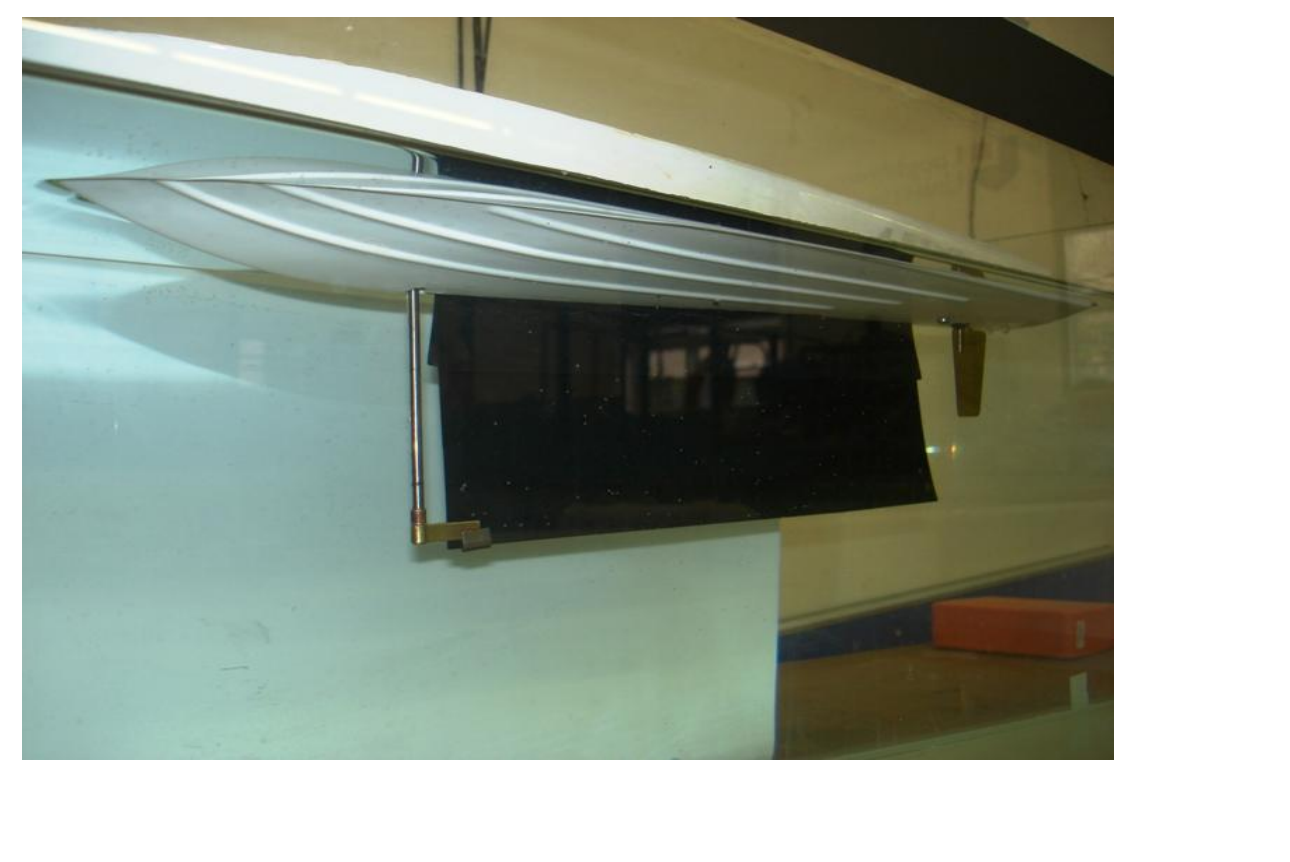

\section{.}

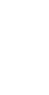

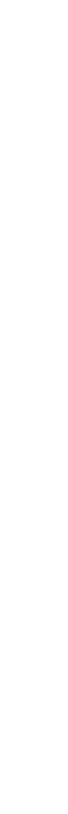

e




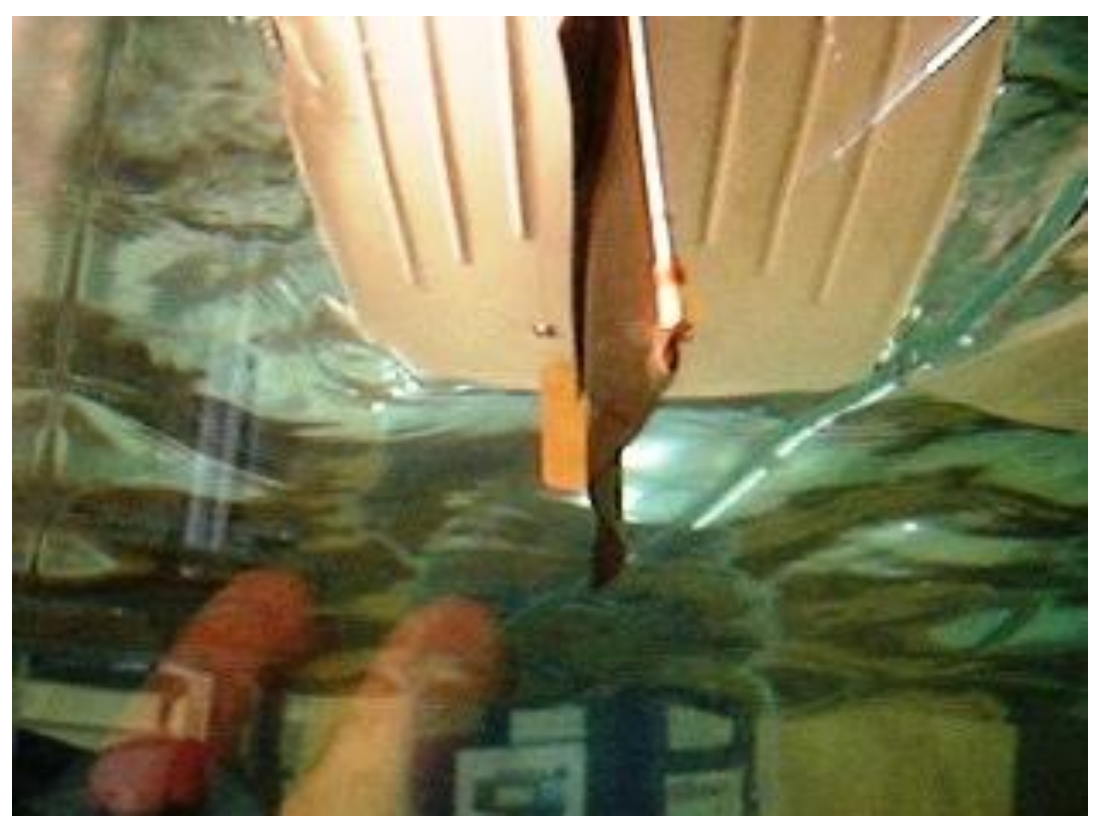

Fig. 5 Underwater view of the hull and of the assembled propulsive plate in action (Krylov et al., 2010); localised flexural wave propagation in the propulsive plate is clearly seen (at $3 \mathrm{~Hz}$ frequency and $20 \mathrm{~mm}$ amplitude). 


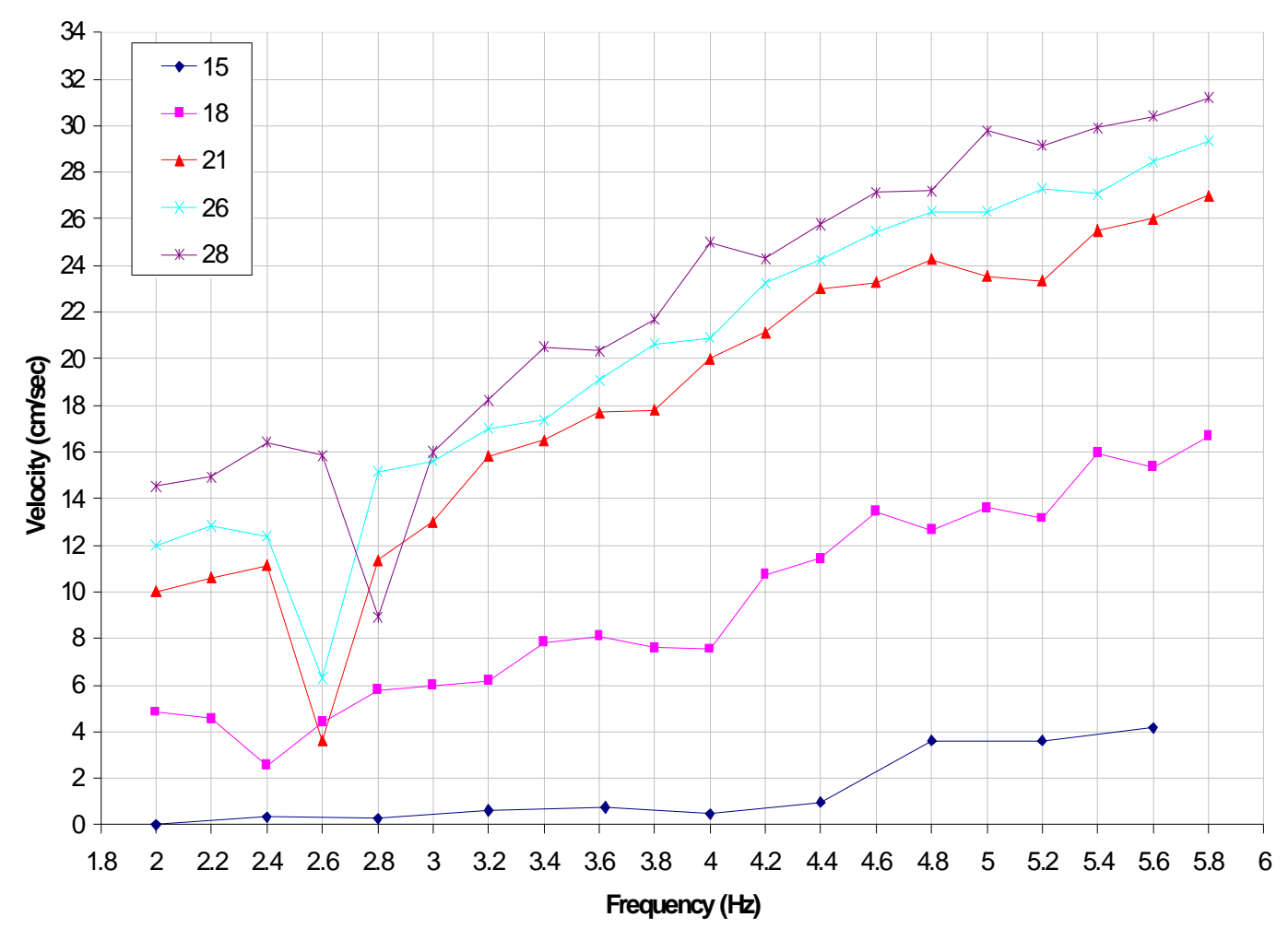

Fig. 6 Experimental values of steady state swimming speed of a model boat as functions of the propulsive wave frequency and amplitude (Krylov et al., 2010). 


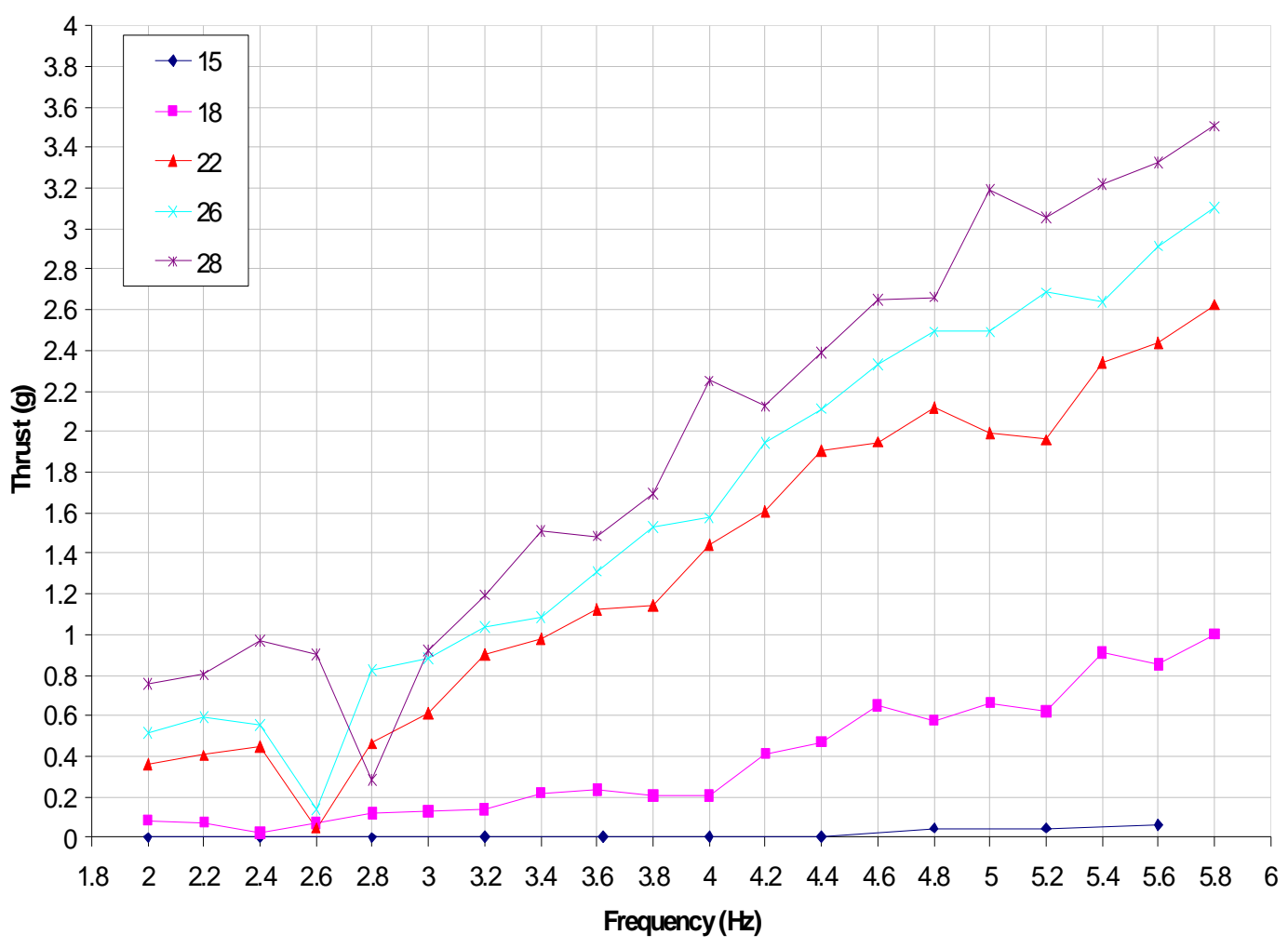

Fig. 7 Experimental values of generated thrust for a model boat as functions of the propulsive wave frequency and amplitude (Krylov et al., 2010). 


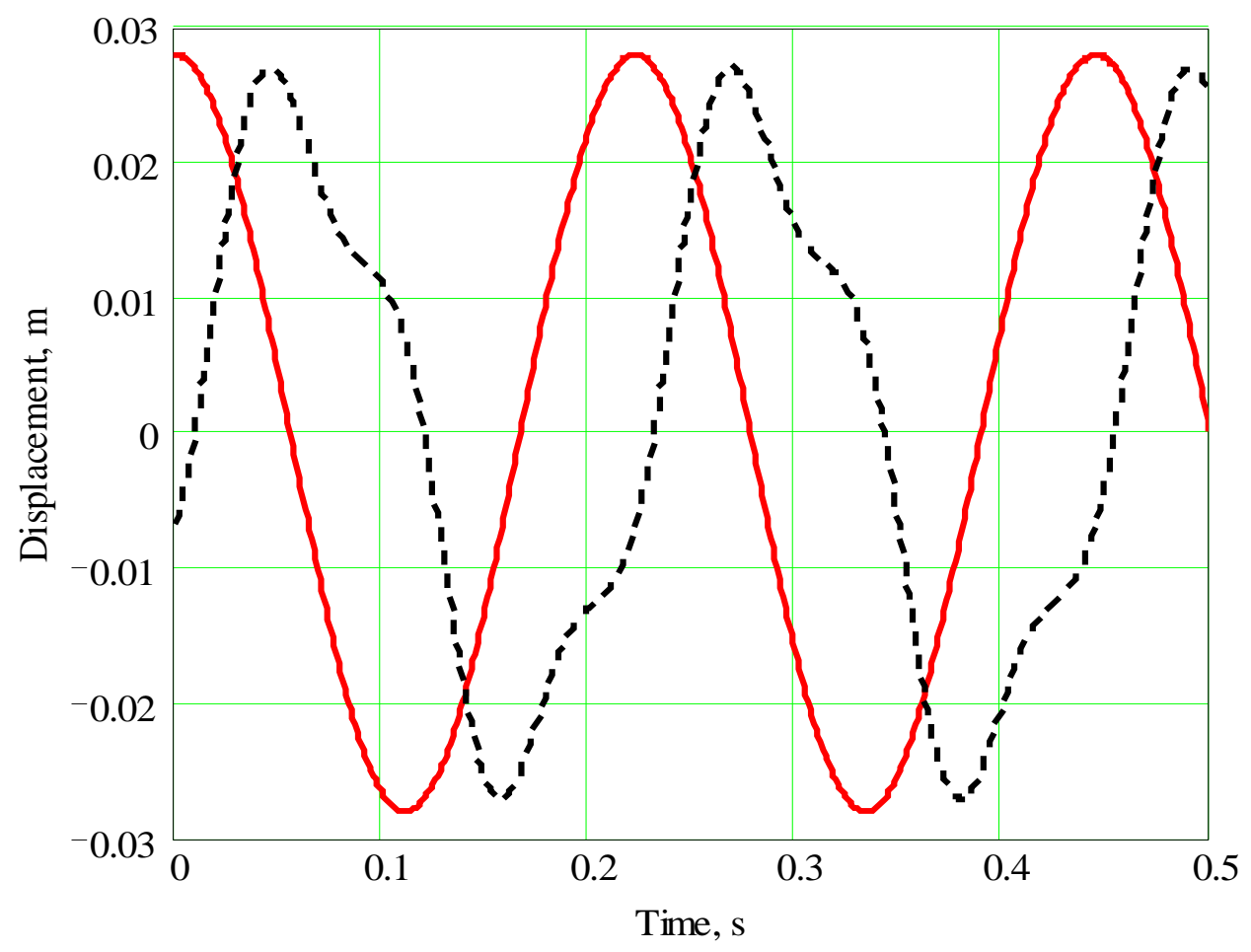

Fig. 8 Calculated waveform distortion of a localised flexural wave due to its nonlinear propagation at frequency $f=4.5 \mathrm{~Hz}$ and amplitude $H=28 \mathrm{~mm}$ along the tip of a rubber wedge immersed in water; calculations have been carried out for $x=0$ (solid line) and for $x=0.17 \mathrm{~m}$ (dashed line). 


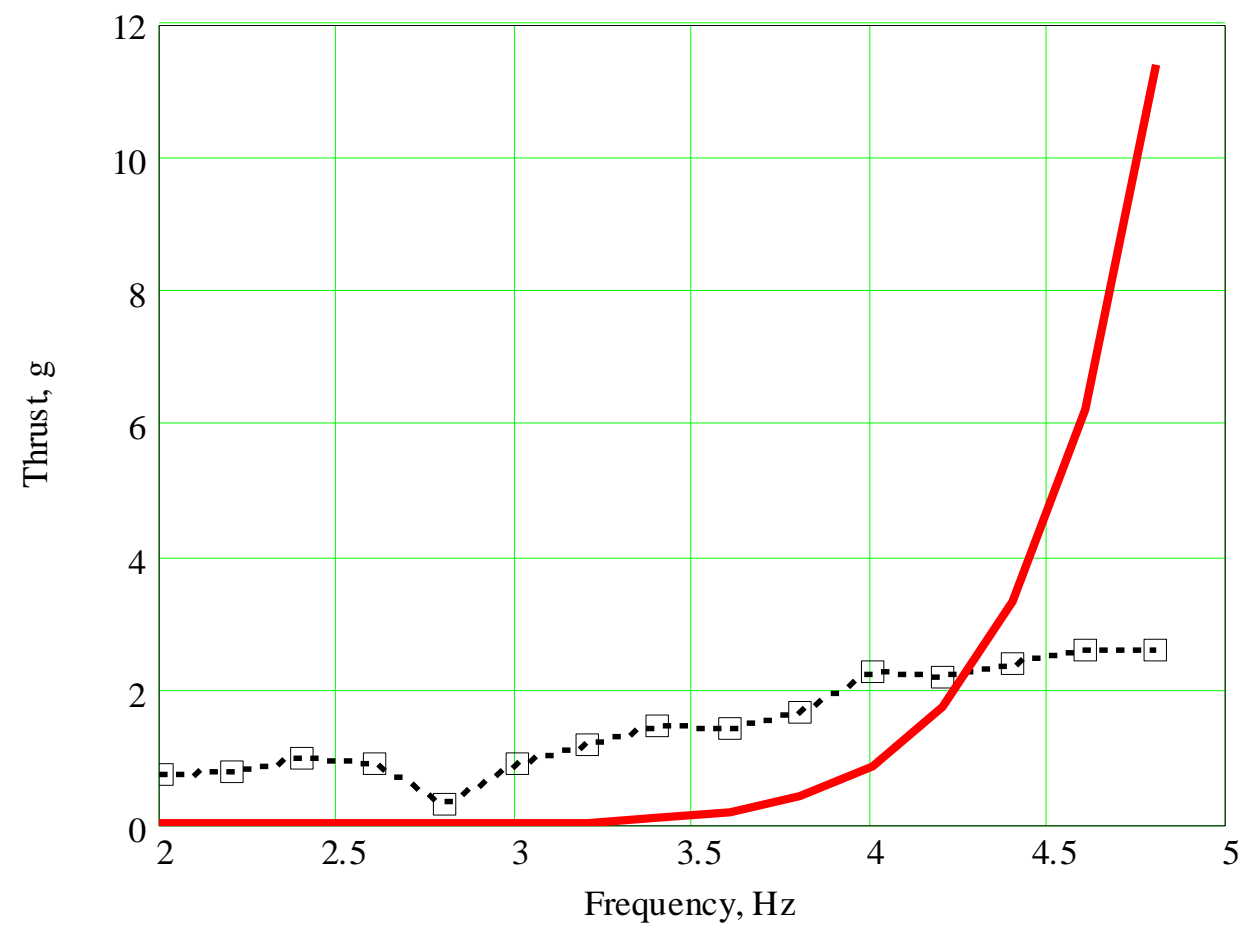

Fig. 9 Calculated thrust as a function of the propulsive wave frequency for the wave amplitude of $28 \mathrm{~mm}$ (solid line) in comparison with the corresponding experimental data (boxes) obtained for a mono-hull model boat (Krylov et al, 2010). 


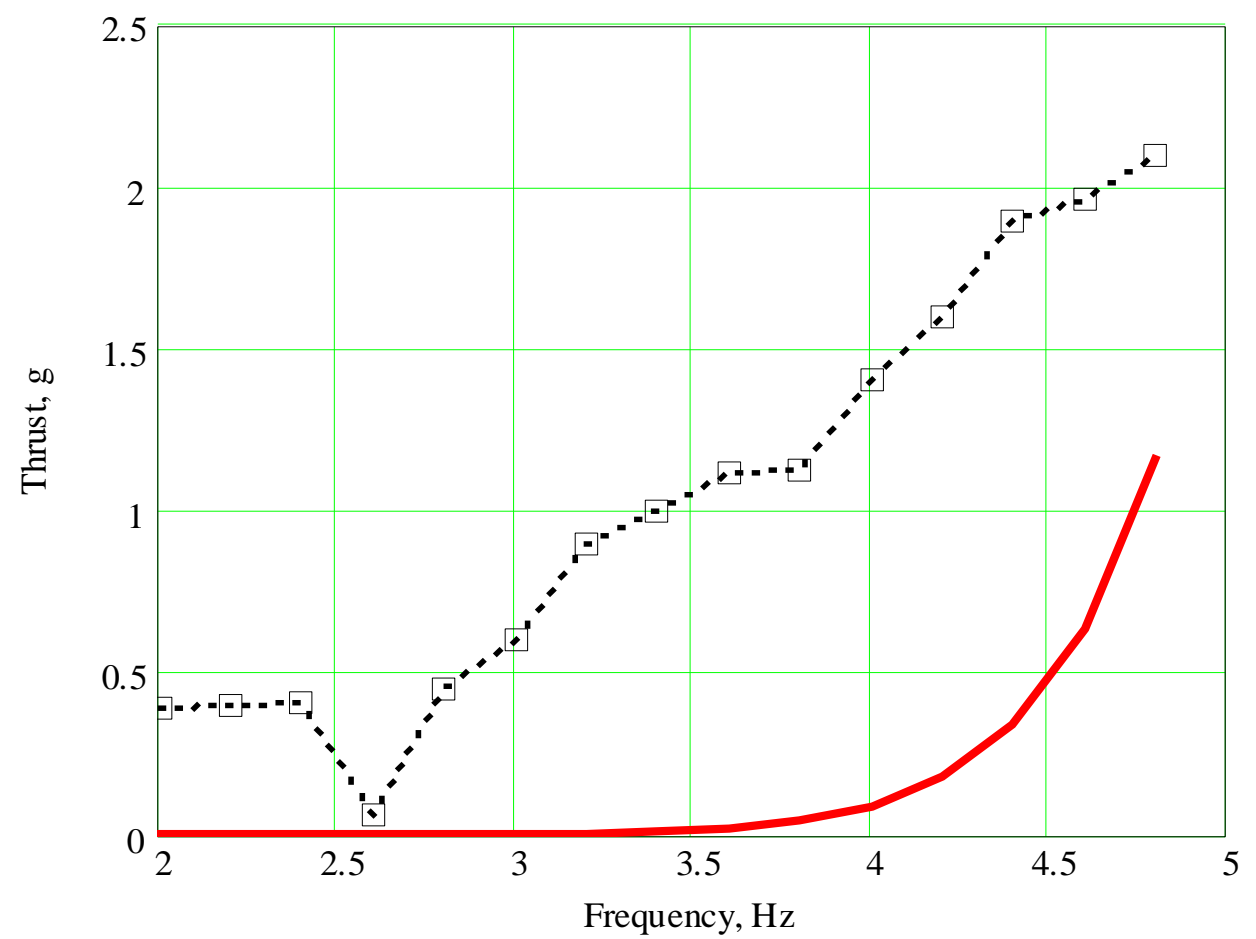

Fig. 10 Calculated thrust as a function of the propulsive wave frequency for the wave amplitude of $22 \mathrm{~mm}$ (solid line) in comparison with the corresponding experimental data (boxes) obtained for a mono-hull model boat (Krylov et al, 2010). 


\section{Tables}

Table 1: Values of the parameters used in calculations

\begin{tabular}{|l|l|l|}
\hline Parameter & Notation & Values \\
\hline Wedge wave displacement amplitude $(\mathrm{m})$ & $H$ & $0.028 ; 0.022$ \\
\hline Wedge angle (degrees) & $\theta$ & 5 \\
\hline Mass density of rubber $\left(\mathrm{kg} / \mathrm{m}^{3}\right)$ & $\rho_{s}$ & 1100 \\
\hline Shear wave speed in rubber $(\mathrm{m} / \mathrm{s})$ & $c_{t}$ & 30 \\
\hline Swimming speed $(\mathrm{m} / \mathrm{s})$ & $U$ & $0.23 ; 0.19$ \\
\hline Propulsive length $(\mathrm{m})$ & $L$ & 0.25 \\
\hline Effective width of fin $(\mathrm{m})$ & $d$ & 0.055 \\
\hline Poisson ratio of rubber & $\sigma$ & 0.49 \\
\hline
\end{tabular}

